\title{
Z-contrast-Microscopy and Density-Functional-Theory Determination of the Atomic Structure of the Fe/AIGaAs Interface and its Impact on Spin Transport
}

\author{
T.J. Zega*, A.T. Hanbicki*, S.C. Erwin*, I. Žutić**, G. Kioseoglou*, C.H. Li*, B.T. Jonker*, and \\ R. M. Stroud*
}

* Naval Research Laboratory, Materials Science and Technology Division, 4555 Overlook Ave SW, Washington, DC 20375

** Department of Physics, State University of New York at Buffalo, Buffalo, NY 14260

High-angle annular-dark-field (HAADF) imaging with a scanning-transmission-electron microscope (STEM) is a promising technique for determining the atomic structure of materials. In principle, HAADF images contain contrast that is dependent on atomic number, but such contrast can deviate from the expected power-law dependence because of variations in sample thickness, defocus value, and delocalization $[1,2]$. Simulated HAADF images can aid interpretation because they allow quantitative comparison with the experimental images.

Information on the structure of a material is a prerequisite for image simulation, and such information is obtainable on materials for which the structure has been previously determined by some other method, e.g., single-crystal X-ray diffraction. Buried interfaces, however, are not amenable to analysis by bulk methods, and so ab initio calculations present a plausible means of predicting energy-minimized interface structures that can be used as the input for image simulations.

Here we combine density-functional theory (DFT) with experimental- and simulated-HAADF imaging to determine the atomic structure of the interface between Fe and AlGaAs in light-emitting diodes used to measure the transport of spin-polarized electrons. Understanding spin transport is fundamental to spin-based electronics (spintronics), a new paradigm for semiconductor electronics in which electron spin, rather than charge, is utilized to carry and store information [3]. Calculations [4] and experiments [5] indicate that spin transport can be strongly influenced by the nature of the interface between the contact and the semiconductor.

Details of sample growth, device fabrication, and spin-transport characterization are reported elsewhere [6]. HAADF images were acquired with a $200 \mathrm{keV}$ JEOL 2200FS TEM. Ground-state interface structures were calculated using DFT [7], and the relaxed atom positions determined from these calculations were used to simulate Z-contrast images with multislice code available in [8].

A HAADF image for an annealed sample with a measured spin polarization of $26 \%$ is shown in Figure 1a. The contrast reveals 0.14-nm periodicities in both Fe and AlGaAs, consistent with their (002) and (004) $d$-spacings, respectively, and shows that the experimental image resolves atomic columns. We compared the experimental image to those simulated from four candidate interface models. We find that the contrast at the interface can be explained by a simple model consisting alternating $\mathrm{Fe}$ and As atoms. Visual comparison of the experimental image and the simulation for the simple model (Fig. 1b) reveals a close match. Nonetheless, we quantitatively assessed the match by measuring and comparing the intensities of the atom columns both parallel and perpendicular to the interface in the experimental and simulated images. We discuss two representative profiles below. 
The intensity profile parallel to the interface (indicated by $\alpha$ to $\alpha^{\prime}$ in Fig. 1a) consists of alternating high- and low-intensity peaks (Fig. 2, solid line). Quantification of the ratios of the peak heights shows that the intensity in the profile follows a $\mathrm{Z}^{1.5}$ to $\mathrm{Z}^{2}$ dependency, consistent with [8], and indicates a row containing alternating Fe and As atoms. The profile from the simulated image (Fig. $2 \mathrm{~b}$, dashed line) of the simple model is in quantitative agreement with the experimental image. A thickness-corrected intensity profile across the interface (indicated by $\beta$ to $\beta^{\prime}$ in Fig. 1a) shows that the columns of Fe, Ga, and As atoms plot as peaks (Fig. 3, red curve). The relative peak intensities and positions are in excellent agreement with those obtained from the DFT-derived simple interface model (Fig. 3, blue curve). We conclude that the interface of this sample is ordered and coherent, with intermixing of the Fe and AlGaAs occurring on a single atomic plane $\left(\alpha-\alpha^{\prime}\right)$, resulting in an interface with alternating Fe and As atoms (Fig. 3, top). HRTEM images from the as-grown form of this sample (18\% spin polarization) indicate a disordered interface extending over approximately 5 atomic planes and preclude assigning a specific interface structure to this sample. We attribute the $44 \%$ increase in spin-injection efficiency of the annealed sample to the greater tunneling efficiency for spin-polarized electrons across the chemically and structurally coherent, annealed interface.

References

[1] L. J. Allen, et al., Ultramicroscopy 96, (2003) 47.

[2] S. D. Findlay, et al., Ultramicroscopy 96, (2003) 65.

[3] G. A. Prinz, Science 27, (1998) 1660.

[4] E. I. Rashba, Phys. Rev. B 62, (2000) R16267.

[5] R. M. Stroud, et al., Phys. Rev. Lett. 89, (2002) 1666021.

[6] B. T. Jonker, Proceedings of the IEEE 91, (2003) 727.

[7] S. C. Erwin, et al., Phys. Rev. B 65, (2002) 205422.

[8] E. J. Kirkland, Advanced computing in electron microscopy (Plenum Press, New York, 1998).

[9] TJZ, IZ, and GK gratefully acknowledge support from the NRL-NRC postdoctoral fellowship program. Research supported in part by ONR, NRL core funds, and the DARPA SpinS program.

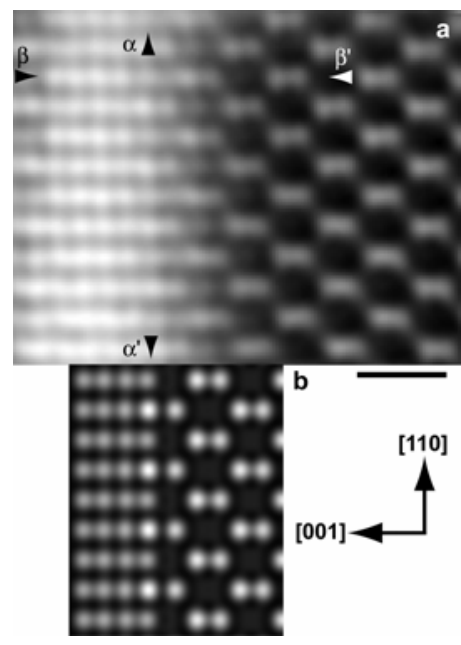

Fig. 1. (a) HAADF image from a sample with $26 \%$ spin polarization. (b) Simulated HAADF image. Scale bar equals $0.5 \mathrm{~nm}$.

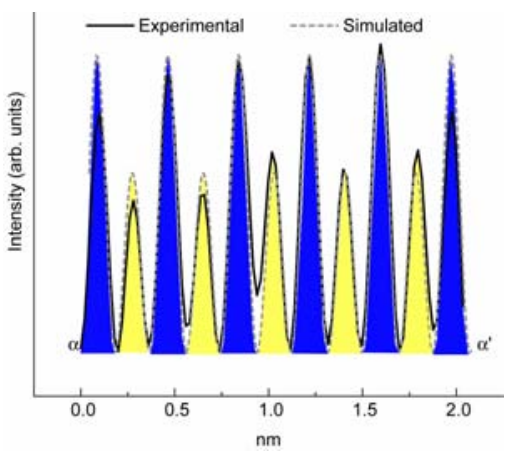

Fig. 2. [110] intensity profile from the positions marked $\alpha$ to $\alpha^{\prime}$ in Fig. 1. Blue peaks correspond to As columns; those in yellow correspond to $\mathrm{Fe}$.

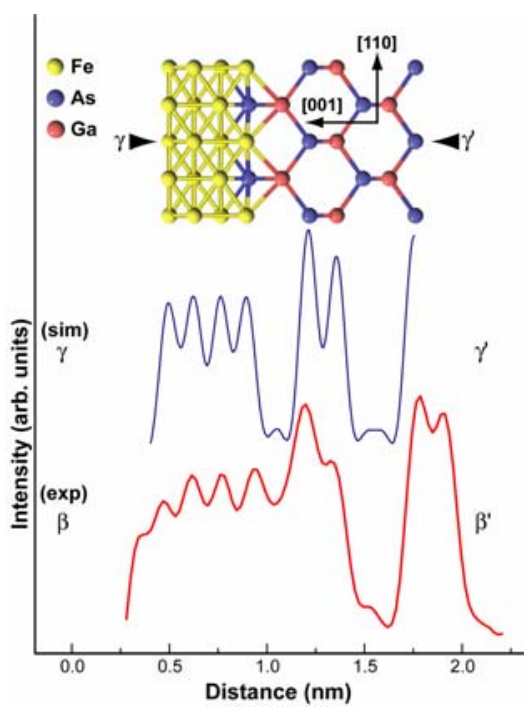

Fig. 3. [001] intensity profiles. Experimental profile shown in red (from the positions marked $b$ to $b^{\prime}$ in Fig. 1); that from the simulation of the simple model (inset) shown in blue. 